\title{
Synthesis of 4,4'-(arylmethylene) bis(3-methyl-1-phenyl-1H-pyrazol-5-ols) and evaluation of their antioxidant and anticancer activities
}

José Eduardo Cadena-Cruz², Luis M. Guamán-Ortiz², Juan Carlos Romero-Benavides³ ${ }^{3}$ Natalia Bailon-Moscoso², Kevin E. Murillo-Sotomayor ${ }^{2}$, Nadia V. Ortiz-Guamán² and Jorge Heredia-Moya ${ }^{*}$

\begin{abstract}
Background: Pyrazoles have attracted particular attention due to the diverse biological activities associated with this heterocyclic system, and some have been shown to be cytotoxic to several human cell lines. Several drugs currently on the market have this heterocycle as the key structural motif, and some have been approved for the treatment of different types of cancer.

Results: 4,4'-(Arylmethylene)bis( 1 H-pyrazol-5-ols) derivatives $\mathbf{3 a - q}$ were synthetized by a three components reaction of 3-methyl-1-phenyl-5-pyrazolone (1) with various benzaldehydes $\mathbf{2}$ catalyzed by sodium acetate at room temperature. The structures of all synthesized compounds were characterized by physicochemical properties and spectral means (IR and NMR) and were evaluated for their radical scavenging activity by DPPH assay and tested in vitro on colorectal RKO carcinoma cells in order to determine their cytotoxic properties. All 4,4'-(arylmethylene)bis $(1 \mathrm{H}$-pyrazol5-ols) derivatives $\mathbf{3 a - q}$ were synthetized in high to excellent yield, and pure products were isolated by simple filtration. All compounds have good radical scavenging activity, and half of them are more active than ascorbic acid used as standard.

Conclusion: Several derivatives proved to be cytotoxic in the RKO cell line. In particular, compound $\mathbf{3 i}$ proved to be a very potent scavenger with an $I C_{50}$ of $6.2 \pm 0.6 \mu \mathrm{M}$ and exhibited an $I C_{50}$ of $9.9 \pm 1.1 \mu \mathrm{M}$ against RKO cell. Autophagy proteins were activated as a survival mechanism, whereas the predominant pathway of death was p53-mediated apoptosis.
\end{abstract}

Keywords: 4,4'-(arylmethylene)bis( $1 \mathrm{H}$-pyrazol-5-ols), Antioxidant, Apoptosis, Autophagy

\section{Introduction}

Heterocycles are common structural units in marketed drugs and in targets in the drug discovery process. Nitrogen-containing rings play an especially important role in

*Correspondence: jorgeh.heredia@ute.edu.ec

${ }^{4}$ Centro de Investigación Biomédica (CENBIO), Facultad de Ciencias de la

Salud Eugenio Espejo, Universidad UTE, 170527 Quito, Ecuador

Full list of author information is available at the end of the article drug development because of their wide variety of therapeutic and pharmacological properties [1]. Pyrazoles and their derivatives have attracted particular attention because they have a wide variety of biological activities $[2,3]$, and several drugs currently on the market, have the pyrazole ring as the key structural motif [4]. Some pyrazole derivatives have been demonstrated to be cytotoxic on several human cell lines [5-8], and, at this time, several drugs that have pyrazoles in their structure have

(c) The Author(s) 2021. This article is licensed under a Creative Commons Attribution 4.0 International License, which permits use, sharing, adaptation, distribution and reproduction in any medium or format, as long as you give appropriate credit to the original author(s) and the source, provide a link to the Creative Commons licence, and indicate if changes were made. The images or other third party material in this article are included in the article's Creative Commons licence, unless indicated otherwise in a credit line to the material. If material is not included in the article's Creative Commons licence and your intended use is not permitted by statutory regulation or exceeds the permitted use, you will need to obtain permission directly from the copyright holder. To view a copy of this licence, visit http://creativeco mmons.org/licenses/by/4.0/. The Creative Commons Public Domain Dedication waiver (http://creativecommons.org/publicdomain/ zero/1.0/) applies to the data made available in this article, unless otherwise stated in a credit line to the data. 
been approved for the treatment of different types of cancer (see Fig. 1).

Edaravone, 3-methyl-1-phenyl-2-pyrazolin-5-one, (1) is a free radical scavenger approved for the treatment of amyotrophic lateral scleorosis (ALS) [9]. The compound is known to have preventive effects on myocardial injury following ischemia and reperfusion in patients with acute myocardial infarction and in brain edema after ischemia and reperfusion injury in animal models and in stroke patients [10]. There are several epidemiological studies related to the incidence of ALS and the development of cancer, that have reported the identification of a set of genes or signaling cascades involved in both diseases [11]. Also, it is well-known that many natural and synthesized antioxidants possessing phenolic hydroxyl groups have improved antioxidant activities by virtue of their abilities to react with free radicals [12], and studies carried out on flavones have shown that there is a relationship between antioxidant and anticancer activity [13].

Michael addition of an aromatic aldehyde 2 with an arylpyrazolone, obtained by the Knoevenagel reaction, allows an easy synthesis of 4,4'-(arylmethylene) bis (1H-pyrazol-5-ols) 3 . These reactions can be done separately $[14,15]$ or in one step, either by a reaction of pseudo-five [16-18] or pseudo-three-components [19]. In practice, most of the reported synthetic routes consist of a one-step condensation of 3-methyl-1-phenyl2-pyrazolin-5-one (1) with different aromatic aldehydes 2 , and most of the reactions used different types of catalyst. These edaravone derivatives incorporating hydroxyl groups in their structures, represent attractive targets for further study, however, only a few reports of biological activity were found in the literature, and the evaluation of compounds with hydroxyl groups is limited to only a few<smiles></smiles>

(non-small cell lung cancer)<smiles>COC1(C(=O)N[C@@H](C)c2ccc(-n3cc(F)cn3)nc2)CCC(c2nc(C)cc(Nc3cc(C)[nH]n3)n2)CC1</smiles>

Pralsetinib (non-small cell lung cancer)<smiles>N#CCC(C1CCCC1)n1cc(-c2ncnc3[nH]ccc23)cn1</smiles>

Ruxolitinib (myelofibrosis)<smiles>Cn1cc(-c2cc3c(N4CCN(c5ncc(C(C)(N)c6ccc(F)cc6)cn5)CC4)ncnn3c2)cn1</smiles>

Avapritinib (gastrointestinal stromal tumors)<smiles>CNc1ccc(-c2ccn(C[C@@H](C)NC(=O)c3cc(CO)[nH]n3)n2)cc1Cl</smiles>

$$
\begin{gathered}
\text { Darolutamide } \\
\text { (prostate cancer) }
\end{gathered}
$$<smiles>COC(=O)N[C@H](C)CNc1nccc(-c2cn(C(C)C)nc2-c2cc(Cl)cc(NS(C)(=O)=O)c2F)n1</smiles>

Encorafenib

(melanoma)

Fig. 1 Approved anti-cancer pyrazole drugs 
examples [20-23], and there is no report of cytotoxicity studies against cancer cells.

\section{Results and discussion Chemistry}

The 4,4'-(arylmethylene)bis( $1 H$-pyrazol-5-ols) 3 were synthesized using $\mathrm{NaOAc}$ as catalyst following the scheme depicted in Scheme 1, using 70\% $\mathrm{EtOH}$ as solvent at room temperature. To find the optimal conditions, the reaction between $\mathbf{2 b}$ and 2 equivalents of $\mathbf{1}$ was chosen as a model, showing $10 \%$ acetate gave the best catalytic effect.

With the optimized conditions, the scope of the reaction was studied using various substituted benzaldehydes $\mathbf{2 a}-\mathbf{q}$ bearing either electron-withdrawing or electron-donating groups to give the corresponding 4,4'-bis-(arilmetilen)bis(1-fenil-3-metil- $1 \mathrm{H}$-pirazol5-ol) derivatives $\mathbf{3 a}-\mathbf{q}$ in good to excellent yield as pure products by simple filtration. The spectroscopic data and melting points of compounds previously reported were in agreement with literature values (see Table 1 and "Experimental" section).

As expected, the time of the reaction with aldehydes bearing electron-withdrawing groups, independent of their location on the ring, were shorter than those with electron-donating groups, except with $\mathbf{2 a}$, which is six times shorter than benzaldehyde (entry 1 ). This could be due to the intramolecular hydrogen bonding in $\mathbf{2 a}$ which enhances the reactivity of the aldehyde. When this hydrogen bond is lost, by protecting or changing the position of the hydroxyl group, the reaction time is higher. However, it is not clear the reason of the reduction of the activity observed in $\mathbf{3 f}$.

\section{Biological activity}

All synthesized compounds were evaluated for antioxidant activity by the $N, N$-diphenyl- $N^{\prime}$-picrylhydrazyl (DPPH) assay as shown in Table 1. All compounds have good radical scavenging activity and half of them are more active than ascorbic acid used as standard. Compound $3 \mathbf{c}$ has the lowest activity $(20.9 \mu \mathrm{M})$ while

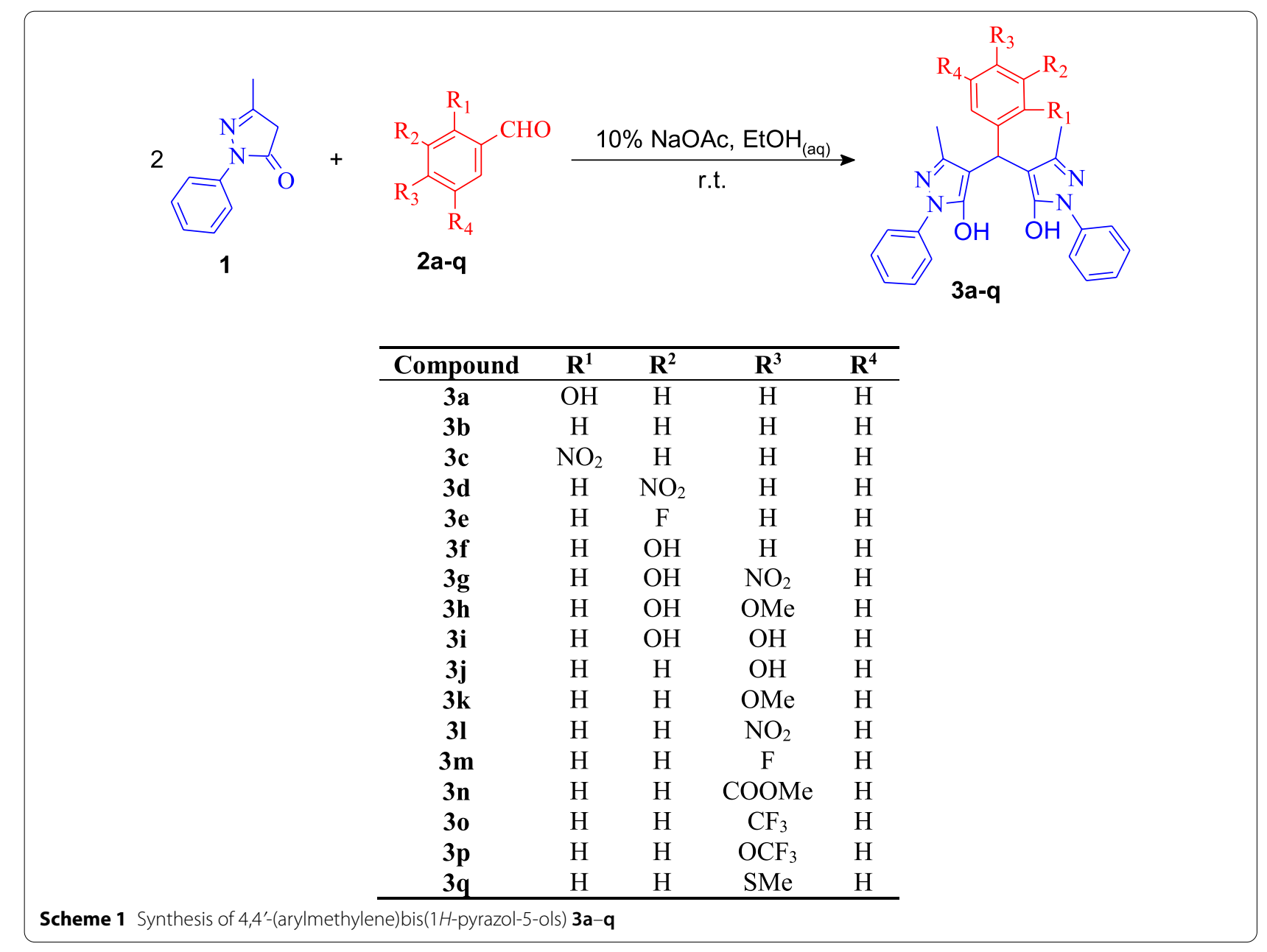


Table 1 Preparation of 4,4'-(arylmethylene)bis(3-methyl-1-phenyl-1 H-pyrazol-5-ols) derivatives 3a-q catalyzed by 10\% NaOAc at room temperature, DPPH scavenging activity and cytotoxic activity against RKO cell line

\begin{tabular}{|c|c|c|c|c|c|}
\hline Compounds & Time (min) & Yield (\%) & $\mathrm{Mp}\left({ }^{\circ} \mathrm{C}\right)$ & $\begin{array}{l}\text { DPPH scavenging } \\
\text { activity } \\
\mathrm{IC}_{50}(\mu \mathrm{M})^{\mathrm{a}}\end{array}$ & $\begin{array}{l}\text { Cytotoxic activity } \\
I_{50}(\mu \mathrm{M})\end{array}$ \\
\hline $3 a$ & 10 & 98 & $219.5-220.6$ & $17.1 \pm 2.5$ & $143.0 \pm 4.9$ \\
\hline $3 b$ & 60 & 97 & $159.5-161.1$ & $14.0 \pm 2.3$ & $105.9 \pm 1.5$ \\
\hline $3 c$ & 10 & 95 & $210.0-211.0$ & $20.9 \pm 5.9$ & $34.1 \pm 4.4$ \\
\hline $3 d$ & 10 & $95^{\mathrm{b}}$ & $150.7-152.0$ & $16.6 \pm 1.1$ & $46.7 \pm 3.3$ \\
\hline $3 e$ & 10 & Quant & $178.0-179.0$ & $13.8 \pm 0.7$ & $36.3 \pm 1.0$ \\
\hline $3 f$ & 480 & $98^{c}$ & $165.0-167.0$ & $19.2 \pm 1.5$ & $84.3 \pm 1.0$ \\
\hline $3 g$ & 40 & 99 & $202.0-204.0$ & $18.7 \pm 1.6$ & $23.5 \pm 5.9$ \\
\hline $3 \mathrm{~h}$ & 180 & $91^{c}$ & $200.0-202.0$ & $13.7 \pm 4.0$ & $97.5 \pm 3.0$ \\
\hline $3 \mathbf{i}$ & 180 & $93^{d}$ & $182.7-184.0$ & $6.2 \pm 0.6^{* * *}$ & $9.9 \pm 1.1$ \\
\hline $3 \mathbf{j}$ & 180 & $97^{c}$ & $217.2-218.9$ & $17.8 \pm 3.7$ & $77.8 \pm 1.1$ \\
\hline $3 k$ & 120 & 92 & $176.0-177.0$ & $19.7 \pm 3.2$ & $89.9 \pm 3.0$ \\
\hline 31 & 20 & 97 & 218.0-219.0 & $18.8 \pm 3.8$ & $27.3 \pm 1.1$ \\
\hline $3 m$ & 60 & 87 & $183.8-185.8$ & $10.2 \pm 0.3$ & $46.4 \pm 1.0$ \\
\hline $3 n$ & 10 & Quant & $217.2-218.7$ & $10.8 \pm 0.3$ & $36.0 \pm 1.0$ \\
\hline 30 & 15 & 96 & $203.0-205.0$ & $9.8 \pm 1.0^{*}$ & $23.6 \pm 1.0$ \\
\hline $3 p$ & 60 & Quant & $174.5-176.0$ & $12.3 \pm 0.9$ & $14.8 \pm 1.0$ \\
\hline $3 q$ & 15 & 60 & $209.1-211.3$ & $13.0 \pm 1.0$ & $40.9 \pm 1.0$ \\
\hline Doxorubicin & - & - & - & - & $2.23 \pm 0.02$ \\
\hline 1 & - & - & - & $18.1 \pm 0.5$ & - \\
\hline Ascorbic acid & - & - & - & $14.0 \pm 2.3$ & - \\
\hline
\end{tabular}

${ }^{a}$ The tests for significance were limited to ANOVA-Dunnett post-test, ${ }^{*} p<0.01,{ }^{* *} p<0.001,{ }^{* * *} p<0.0001$ vs. 1

${ }^{\mathrm{b}}$ Using $100 \% \mathrm{EtOH}$

' Using $60 \%$ EtOH

${ }^{d}$ Using $50 \% \mathrm{EtOH}$

compound $3 \mathbf{i}$ proved to be a very potent scavenger with an $\mathrm{IC}_{50}$ of $6.2 \mu \mathrm{M}$. In fact, most bispyrazoles had better radical scavenging activity than $\mathbf{1}$, and only $\mathbf{3 c}, \mathbf{3 f}, \mathbf{3 k}$, and $\mathbf{3 l}$ are less potent scavengers.

Theoretical calculation of $\mathbf{1}$ shows that an $\mathrm{H}$-atom abstraction rather than electron-transfer reaction is involved in the radical-scavenging process [24, 25]. Because in structure 3 the enol tautomer is more stable than the keto form, it would be expected that this hydrogen abstraction occurs on the hydroxyl group. Theoretical calculations of the enol tautomer of $\mathbf{1}$ show lower dissociation energy of this bond, so the abstraction of this hydrogen would be the most important for the scavenger properties of the edaravone [26].

The higher antioxidant activity of $3 \mathbf{i}$ could suggest that the abstraction of the phenolic hydrogens instead of the enolic hydrogens would be more important for the stabilization of radicals, since the presence of the orthodihydroxy system is known to increase the stabilization of radicals [27]. This stabilization, provided by intramolecular hydrogen bonding in the radical formed has been confirmed by theoretical calculation in catechol derivatives [28]. Due to steric factors, the structure of 3 is not coplanar. Nonetheless, the results suggest that the conjugation of the radical is not restricted only to the pyrazol rings, since a contribution in the stabilization of the radical due to the aryl moiety is observed. Furthermore, both electron-donating and electron-withdrawing groups stabilize the radical, however, it is not clear how this stabilization occurs.

All derivatives were tested in vitro on colorectal RKO carcinoma cells in order to determine their cytotoxic properties (Table 1). Cells were exposed to each compound at five increasing concentrations for $48 \mathrm{~h}$ and their viability was monitored through MTS assay; as expected, dose-dependent effects were observed. For compounds $3 \mathbf{a}$ and $\mathbf{3 b}$ the observed $\mathrm{IC}_{50}$ was greater than $100 \mu \mathrm{M}$, however, the vast majority are below $50 \mu \mathrm{M},(3 \mathbf{c}, \mathbf{3 d}, \mathbf{3 e}$, 3g, 3i, 31-3q), the compound with the highest cytotoxic activity was $3 \mathbf{i}$ with an $\mathrm{IC}_{50}$ of $9.9 \mu \mathrm{M}$.

The MTS test is a proliferation test, but it does not distinguish between cytostatic and cytotoxic effect. To understand how the most powerful compound of the derivatives is acting, in this case $\mathbf{3} \mathbf{i}$, the trypan blue dye 
exclusion test was performed at 24 and $48 \mathrm{~h}$. As indicated in Fig. 2, both cell numbers are seen to decrease in a dose-dependent manner (Fig. 2A); observing a decrease in the number of cells that could be related to a cytostatic effect. Thus, a decrease in cell viability related to the cytotoxic effect is also observed (Fig. 2B), the same as they agree with the morphological changes observed in Fig. 2C.

Studied with the proteins involved in both apoptosis and autophagia, and p53 and p21 proteins controlling cell proliferation and death response were carried out [29].
Since cell death pathways must be examined prior to the morphological changes, a shorter time $(24 \mathrm{~h})$ and higher doses were chosen $(30,40$ and $50 \mu \mathrm{M})$. Figure $3 \mathrm{~A}$ shows that p53 increases significatively in a dose-dependent manner, indicating that this protein could be involved in the induced cell death process [30] by compound $3 \mathbf{i}$. A similar effect was observed in A2780 (ovarian adenocarcinoma), P388 (leukemia), and A549 (lung carcinoma) human cell lines [31,32] after treatment with pyrazole derivatives. One of the major targets for $\mathrm{p} 53$ is $\mathrm{p} 21$, a protein related to cell cycle arrest; according to our results,

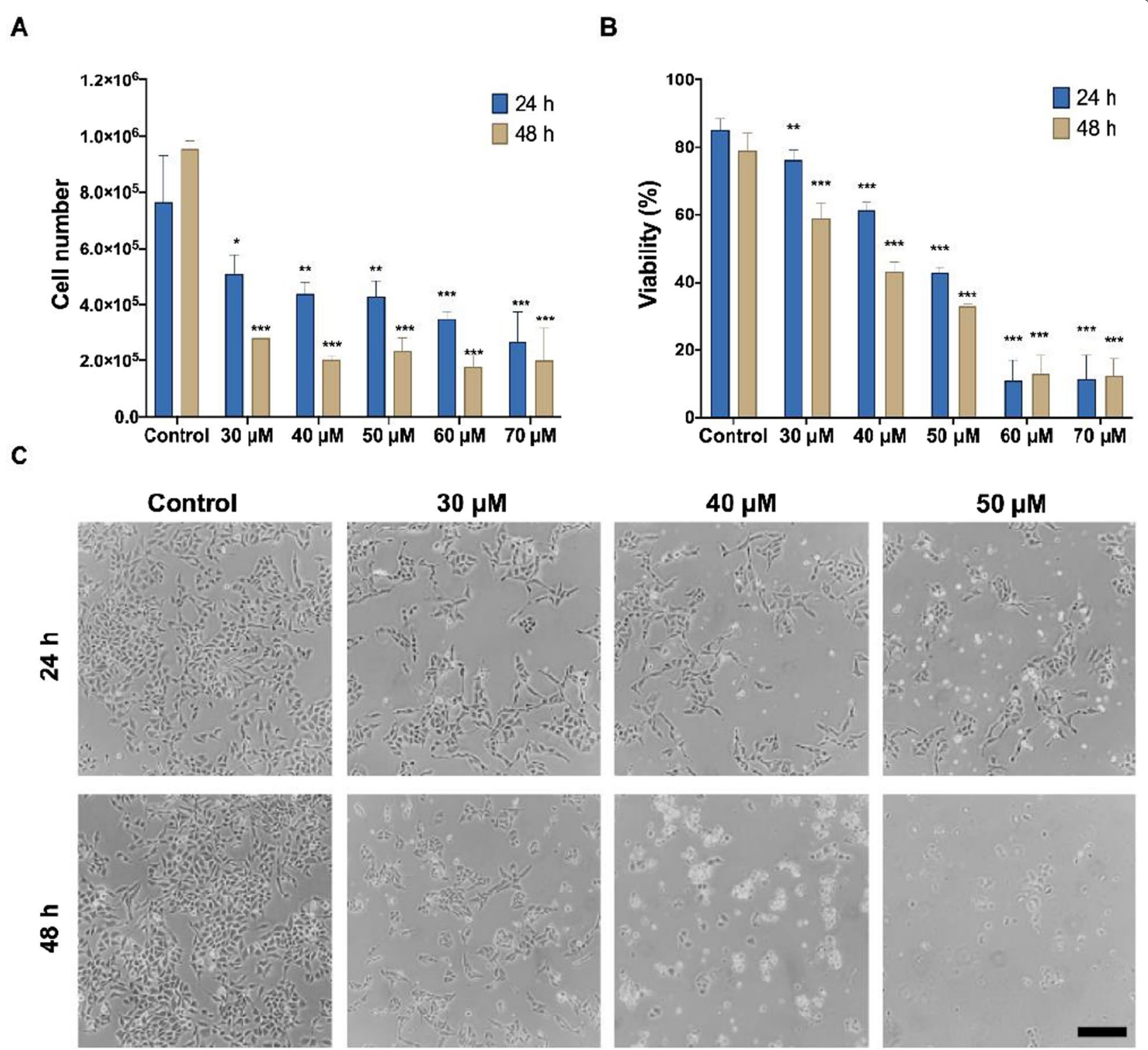

Fig. 2 Cytotoxic effect of compound 3i. RKO cell line were exposed to 10-70 $\mu \mathrm{M}$ of $\mathbf{3 i}$ for 24 and $48 \mathrm{~h}$ and evaluated through Trypan Blue dye exclusion assay. A cell population, and $\mathbf{B}$ percentages of cell viability. The tests for significance were limited to ANOVA-Dunnett post-test, ${ }^{*} p<0.01$, ${ }^{* *} p<0.001,{ }^{* * *} p<0.0001$ vs. control. C Cell morphology after exposure at 30, 40, and $50 \mu \mathrm{M}$; Horizontal bar in bottom figure $=50 \mu \mathrm{m}$ 


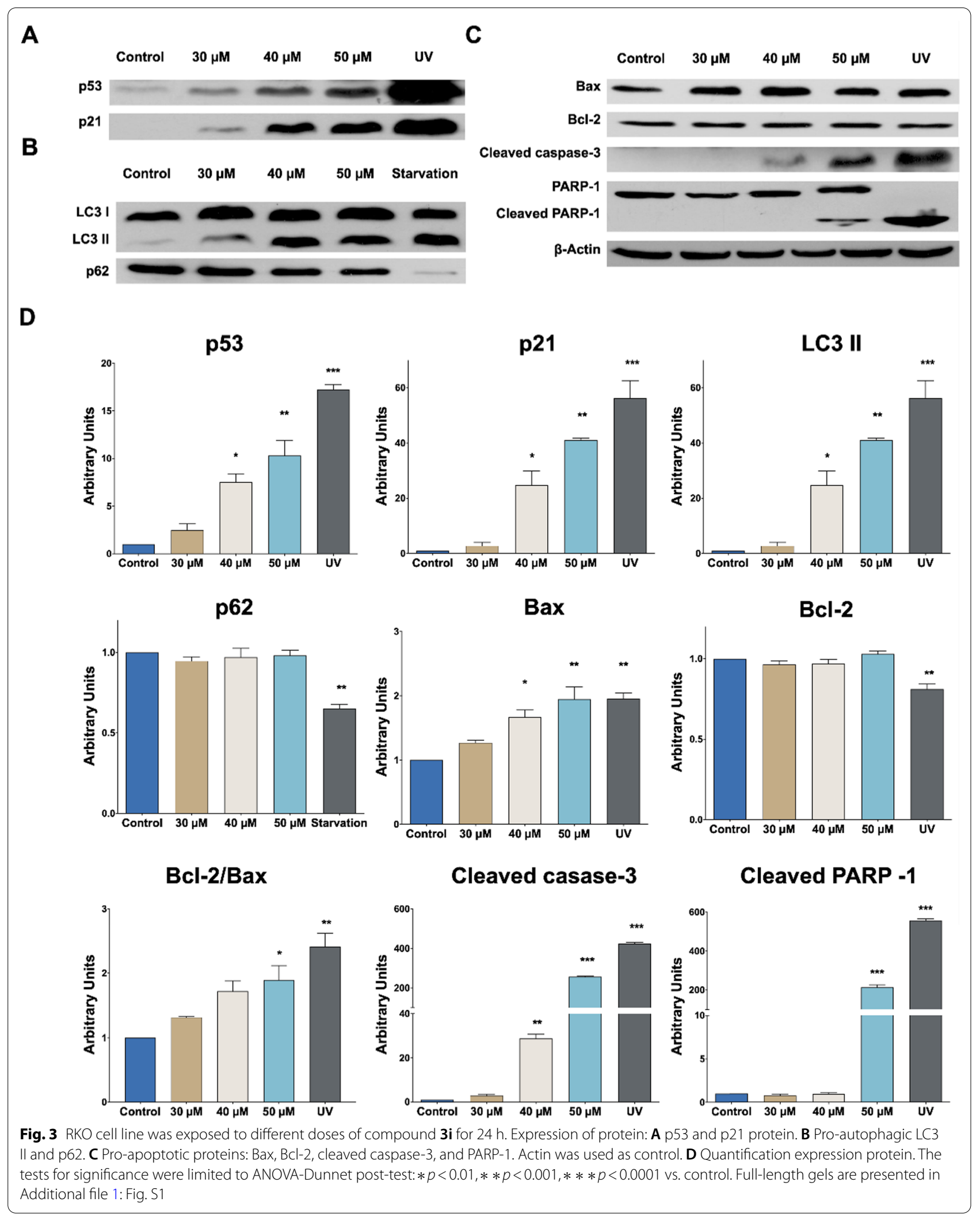


p21 increases significantly in all doses tested (Fig. 3A, D); explaining therefore the inhibition of cell grow (cytostatic effect).

Autophagy pathway activation has been found to be induced by pyrazol derivatives in A549 lung cancer cell [33]. Therefore, pro-autophagic proteins were also studied (Fig. 3B, D). LC3 I is converted into its active form LC3 II at the beginning of the autophagy, while p62 is degraded in the last phase of this cell death pathway [34]. Our results showed a significant increase in LC3 II in the highest concentration, whereas no changes in p62 were detected. Accordingly, in our study, compound $3 \mathbf{i}$ is able to induce autophagy as a protective mechanism [35]

It is well-known that p53 upregulates Bax to promote the intrinsic apoptosis pathway, which ends in the activation of caspase-3 [30]. Consequently, a significant increase in Bax upregulation was detected, together with the caspase- 3 activation mostly in the highest concentration; whereas no changes were observed in the regulation of the anti-apoptotic protein Bcl-2 (Fig. 3C, D). This later protein has been found to interact with Beclin-1 to form the Bcl-2-Beclin-1 complex, which inhibits autophagy and allows the activation of apoptosis [36], thus explaining the observation that there is no variation in Bcl-2 expression and the lack of activation of autophagy. Moreover, a significant increase in $\mathrm{Bax} / \mathrm{Bcl}-2$ ratio was observed; indicating therefore that the RKO cell line is susceptible to apoptosis after treatment [37]. One of the multiple substrates of caspase-3 is PARP-1, a well-known apoptotic biomarker, which was also found significantly cleaved in the highest concentration (Fig. 3C, D). All these findings therefore show that compound $3 \mathbf{i}$ is able to induce apoptosis on RKO cell line, mediated by p53.

Finally, the analysis of the substituents shows that compounds bearing electron-withdrawing groups, independent of their location on the ring, are more active than compounds with electron-donating substituents, except for $3 \mathbf{i}\left(\mathrm{IC}_{50}=9.9 \pm 1.1 \mu \mathrm{M}\right)$ which is the most active compound despite having two hydroxyl groups. The ortho position of these hydroxyls would be responsible for the high activity observed since it has been reported that catechol compounds with hydroxyl residues ortho to each other are susceptible to oxidation leading to cell apoptosis, mainly due to generation of quinone through autoxidation and subsequent induction of p53 and caspase- 3 activation [38].

\section{Conclusion}

In this work, 4,4'-(arylmethylene)bis(3-methyl-1-phenyl-1 $H$-pyrazol-5-ols) $\mathbf{3 a - q}$ were synthesized at room temperature using $\mathrm{NaOAc}$ as a catalyst in high to excellent yields and pure products were isolated by simple filtration. Most compounds show better DPPH radical scavenging activities than edaravone 1, and some compounds show moderate cytotoxicity against RKO cell. In both activities the most potent compound was $\mathbf{3 i}$, with cytotoxic activity to the RKO cell line present in a dose and time-depended manner. Indeed, this compound was able to induce the apoptotic cell death pathway.

\section{Experimental \\ Chemistry}

All solvents and reagents were from Sigma Aldrich and used without further purification. All melting points are uncorrected and were determined on a Büchi Melting Point M-560 apparatus. FTIR spectra were recorded by a Perkin Elmer FTIR Spectrum One by using ATR system $\left(4000-650 \mathrm{~cm}^{-1}\right)$. The ${ }^{1} \mathrm{H}$ and ${ }^{13} \mathrm{C}$ NMR spectra were recorded at $298 \mathrm{~K}$ on a JEOL ECA $400 \mathrm{MHz}$ or Bruker Advance $500 \mathrm{MHz}$ spectrometer equipped with a $\mathrm{z}$-gradient, triple-resonance $\left({ }^{1} \mathrm{H},{ }^{13} \mathrm{C},{ }^{15} \mathrm{~N}\right)$ cryoprobe, using DMSO-d6 as solvent. The ${ }^{19} \mathrm{~F}-\mathrm{NMR}$ spectra were acquired on an Oxford Instruments Pulsar benchtop NMR $60 \mathrm{MHz}$ Spectrometer. Chemical shifts are expressed in ppm with tetramethylsilane (TMS, $\delta=0 \mathrm{ppm}$ ) as an internal reference for protons and trifluoroacetic acid (TFA, $\delta=-75.39 \mathrm{ppm}$ ) for fluorine. Accurate mass data were obtained using a Waters (Waltham, MA) model LCT Premiere time-of-flight (TOF) mass spectrometer. Reactions were monitored by TLC on silica gel using ethyl acetate/hexane mixtures as a solvent and compounds visualized by UV lamp. The reported yields are for the purified material and are not optimized.

\section{General procedure for the synthesis of 4,4'-(arylmethylene) bis(3-methyl-1-phenyl-1H-pyrazol-5-ols) 3a-q}

To a solution of $0.4 \mathrm{mmol}$ aldehyde $2 \mathbf{a}-\mathbf{q}$ and $0.8 \mathrm{mmol}$ pyrazole 1 in $4 \mathrm{~mL}$ of $70 \% \mathrm{EtOH}$ at room temperature, $40.2 \mu \mathrm{L}$ of $1 \mathrm{M} \mathrm{NaOAc}$ were added and the mixture was stirred until the reaction was complete (see Table 1). Water was added to obtain $50 \% \mathrm{EtOH}$ and the mixture was filtered, washed with $50 \% \mathrm{EtOH}$ and dried to obtain pure product.

4,4'-[(2-Hydroxyphenyl)methylene]bis(3-methyl-1-phenyl-1H-pyrazol-5-ol) $3 a$ Yield $98 \%$ as a white solid; mp 219.5-220.6 ${ }^{\circ} \mathrm{C}$ [Lit. 218-220 [39]]; ${ }^{1} \mathrm{H}-\mathrm{NMR}$ (400 MHz, DMSO-d ) $\delta: 14.30$ (br. s., $1 \mathrm{H}, \mathrm{OH}$ ), 12.38 (br. s., 1 $\mathrm{H}, \mathrm{OH}), 9.51$ (br. s., $1 \mathrm{H}, \mathrm{OH}), 7.70$ (d, $J=8.0 \mathrm{~Hz}, 4 \mathrm{H}$, $\mathrm{Ar}-\mathrm{H}), 7.56$ (d, $J=7.6 \mathrm{~Hz}, 1 \mathrm{H}, \mathrm{Ar}-\mathrm{H}), 7.43(\mathrm{t}, J=7.6 \mathrm{~Hz}$, $4 \mathrm{H}, \mathrm{Ar}-\mathrm{H}), 7.24(\mathrm{t}, J=6.7 \mathrm{~Hz}, 2 \mathrm{H}, \mathrm{Ar}-\mathrm{H}), 6.97(\mathrm{t}$, $J=7.6 \mathrm{~Hz}, 1 \mathrm{H}, \mathrm{Ar}-\mathrm{H}), 6.75(\mathrm{~d}, J=7.9 \mathrm{~Hz}, 1 \mathrm{H}, \mathrm{Ar}-\mathrm{H})$, 
$6.71(\mathrm{t}, J=7.6 \mathrm{~Hz}, 1 \mathrm{H}, \mathrm{Ar}-\mathrm{H}), 5.18(\mathrm{~s}, 1 \mathrm{H}, \mathrm{CH}), 2.29(\mathrm{~s}$, $\left.6 \mathrm{H}, \mathrm{CH}_{3}\right)$.

4,4'-(Phenylmethylene)bis(3-methyl-1-phenyl-1H-pyrazol-5-ol) $3 b$ Yield $97 \%$ as a white solid; mp 159.5161. ${ }^{\circ} \mathrm{C}$ [Lit. 161-163 [40]]; ${ }^{1} \mathrm{H}-\mathrm{NMR}(400 \mathrm{MHz}$, DMSO$d_{6}$ ) $\delta: 13.94$ (br. s., $\left.1 \mathrm{H}, \mathrm{OH}\right), 12.44$ (br. s., $\left.1 \mathrm{H}, \mathrm{OH}\right), 7.71$ $(\mathrm{d}, J=7.9 \mathrm{~Hz}, 4 \mathrm{H}, \mathrm{Ar}-\mathrm{H}), 7.44(\mathrm{t}, J=7.9 \mathrm{~Hz}, 4 \mathrm{H}, \mathrm{Ar}-\mathrm{H})$, 7.31-7.21 (m, $6 \mathrm{H}, \mathrm{Ar}-\mathrm{H}), 7.20-7.14(\mathrm{~m}, 1 \mathrm{H}, \mathrm{Ar}-\mathrm{H})$, 4.96 (s, $1 \mathrm{H}, \mathrm{CH}$ ), 2.32 (br. s., $6 \mathrm{H}, \mathrm{CH}_{3}$ ).

4,4'-[(2-Nitrophenyl)methylene]bis(3-methyl-1-phenyl-1H-pyrazol-5-ol) $3 c$ Yield $95 \%$ as a yellow solid; $\mathrm{mp}$ 210.0-211.0 ${ }^{\circ} \mathrm{C}$ [Lit. 218-220 [41]]; ${ }^{1} \mathrm{H}-\mathrm{NMR}(400 \mathrm{MHz}$, DMSO-d $_{6}$ ) $\delta: 13.35$ (br. s., $\left.1 \mathrm{H}, \mathrm{OH}\right), 12.60$ (br. s., $1 \mathrm{H}$, $\mathrm{OH}), 7.72(\mathrm{~d}, J=7.9 \mathrm{~Hz}, 1 \mathrm{H}, \mathrm{Ar}-\mathrm{H}), 7.66(\mathrm{~d}, J=7.9 \mathrm{~Hz}, 4$ $\mathrm{H}, \mathrm{Ar}-\mathrm{H}), 7.62(\mathrm{~m}, 2 \mathrm{H}, \mathrm{Ar}-\mathrm{H}), 7.48(\mathrm{~m}, 1 \mathrm{H}, \mathrm{Ar}-\mathrm{H}), 7.43$ $(\mathrm{t}, J=7.9 \mathrm{~Hz}, 4 \mathrm{H}, \mathrm{Ar}-\mathrm{H}), 7.25(\mathrm{t}, J=7.3 \mathrm{~Hz}, 2 \mathrm{H}, \mathrm{Ar}-\mathrm{H})$, $5.43(\mathrm{~s}, 1 \mathrm{H}, \mathrm{CH}), 2.24$ (br. s., $\left.6 \mathrm{H}, \mathrm{CH}_{3}\right)$.

4,4'-[(3-Nitrophenyl)methylene]bis(3-methyl-1-phenyl-1H-pyrazol-5-ol) $3 d$ Yield $95 \%$ as a white solid; mp 150.7-152.0 ${ }^{\circ} \mathrm{C}$ [Lit. 150-152 [42]]; ${ }^{1} \mathrm{H}-\mathrm{NMR}(400 \mathrm{MHz}$, $D M S O-d_{6}$ ) $\delta: 13.87$ (br. s., $\left.1 \mathrm{H}, \mathrm{OH}\right), 12.64$ (br. s., $1 \mathrm{H}$, $\mathrm{OH}), 8.09$ (s, $1 \mathrm{H}, \mathrm{Ar}-\mathrm{H}), 8.08$ (d, J=8.6 Hz, $1 \mathrm{H}, \mathrm{Ar}-\mathrm{H})$, $7.74(\mathrm{~d}, J=7.9 \mathrm{~Hz}, 1 \mathrm{H}, \operatorname{Ar}-\mathrm{H}), 7.70(\mathrm{~d}, J=8.5 \mathrm{~Hz}, 4$ $\mathrm{H}, \mathrm{Ar}-\mathrm{H}), 7.61(\mathrm{t}, J=8.6,7.9 \mathrm{~Hz}, 1 \mathrm{H}, \mathrm{Ar}-\mathrm{H}), 7.45(\mathrm{t}$, $J=7.9 \mathrm{~Hz}, 4 \mathrm{H}, \mathrm{Ar}-\mathrm{H}), 7.26(\mathrm{~d}, J=7.4 \mathrm{~Hz}, 2 \mathrm{H}, \mathrm{Ar}-\mathrm{H})$, 5.15 (s, $1 \mathrm{H}, \mathrm{CH}$ ), 2.35 (br. s., $6 \mathrm{H}, \mathrm{CH}_{3}$ ).

4,4'-[(3-Fluorophenyl)methylene]bis(3-methyl-1-phenyl-1H-pyrazol-5-ol) $3 e$ Yield quantitative as a white solid; mp 178.0-179.0 ${ }^{\circ} \mathrm{C}$ [Lit. 183-184 [40]]; ${ }^{1} \mathrm{H}-\mathrm{NMR}$ $\left(500 \mathrm{MHz}, D M S O-d_{6}\right) \delta: 7.70(\mathrm{~d}, J=8.0 \mathrm{~Hz}, 4 \mathrm{H}, \mathrm{Ar}-\mathrm{H})$, $7.44(\mathrm{t}, J=7.8 \mathrm{~Hz}, 4 \mathrm{H}, \mathrm{Ar}-\mathrm{H}), 7.29-7.36(\mathrm{~m}, 1 \mathrm{H}, \mathrm{Ar}-\mathrm{H})$, 7.21-7.28 (m, 2H, Ar-H), $7.10(\mathrm{~d}, J=8.2 \mathrm{~Hz}, 1 \mathrm{H}, \mathrm{Ar}-\mathrm{H})$, 6.98-7.04 (m, 2H, Ar-H), 4.97 (s, 1H, CH), 2.31 (br. s., $\left.6 \mathrm{H}, \mathrm{CH}_{3}\right)$.

4,4'-[(3-Hydroxyphenyl)methylene]bis(3-methyl-1-phenyl-1H-pyrazol-5-ol) $3 f$ Yield $98 \%$ as a white solid; $\mathrm{mp}$ 165.0-167.0 ${ }^{\circ} \mathrm{C}$ [Lit. 164-166 [43]]; ${ }^{1} \mathrm{H}-\mathrm{NMR}(400 \mathrm{MHz}$, DMSO-d $\left.d_{6}\right) \delta: 13.95$ (br. s., $\left.1 \mathrm{H}, \mathrm{OH}\right), 9.21(\mathrm{~s}, 1 \mathrm{H}, \mathrm{Ar}-\mathrm{H})$, $7.71(\mathrm{~d}, J=7.9 \mathrm{~Hz}, 4 \mathrm{H}, \mathrm{Ar}-\mathrm{H}), 7.44(\mathrm{t}, J=7.8 \mathrm{~Hz}, 4 \mathrm{H}$, Ar-H), 7.24 (t, $J=7.3 \mathrm{~Hz}, 2 \mathrm{H}, \mathrm{Ar}-\mathrm{H}), 7.04(\mathrm{t}, J=7.8 \mathrm{~Hz}$, $1 \mathrm{H}, \mathrm{Ar}-\mathrm{H}), 6.68$ (br. s, $1 \mathrm{H}, \mathrm{OH}), 6.65(\mathrm{~d}, J=7.7 \mathrm{~Hz}, 1 \mathrm{H}$, Ar-H), 6.55 (dd, J=7.9, $1.5 \mathrm{~Hz}, 1 \mathrm{H}, \mathrm{Ar}-\mathrm{H}), 4.86(\mathrm{~s}, 1 \mathrm{H})$, 2.30 (br. s., $6 \mathrm{H})$.

4, 4'-[(3-Hydroxy-4-nitrophenyl)methylene] bis(3-methyl-1-phenyl-1H-pyrazol-5-ol) $3 g$ Yield 99\% as a yellow solid; mp $202.0-204.0{ }^{\circ} \mathrm{C}$ (d); FTIR $\left(\mathrm{cm}^{-1}\right)$ : $3616(\mathrm{OH}), 1604(\mathrm{C}=\mathrm{C}), 1600(\mathrm{C}=\mathrm{N}), 1577\left(\mathrm{NO}_{2}\right)$,
$1499(\mathrm{C}=\mathrm{C}), 1353(\mathrm{OH}), 1331\left(\mathrm{NO}_{2}\right), 1233(\mathrm{OH}), 1120$ $(\mathrm{C}-\mathrm{OH}) ;{ }^{1} \mathrm{H}-\mathrm{NMR}\left(500 \mathrm{MHz}, \mathrm{DMSO}-d_{6}\right) \delta$ : 13.84 (br. s., $1 \mathrm{H}, \mathrm{OH}), 10.84(\mathrm{~s}, 1 \mathrm{H}, \mathrm{OH}), 7.83(\mathrm{~d}, J=8.2 \mathrm{~Hz}, 1 \mathrm{H}$, $\mathrm{Ar}-\mathrm{H}), 7.71(\mathrm{~d}, J=8.2 \mathrm{~Hz}, 4 \mathrm{H}, \mathrm{Ar}-\mathrm{H}), 7.45(\mathrm{t}, J=8.0 \mathrm{~Hz}$, $4 \mathrm{H}, \mathrm{Ar}-\mathrm{H}), 7.26(\mathrm{t}, J=7.4 \mathrm{~Hz}, 2 \mathrm{H}, \mathrm{Ar}-\mathrm{H}), 7.04(\mathrm{~s}, 1 \mathrm{H}$, Ar-H), 6.81 (dd, $J=1.9,8.5 \mathrm{~Hz}, 1 \mathrm{H}, \mathrm{Ar}-\mathrm{H}), 5.00(\mathrm{~s}, 1 \mathrm{H}$, $\mathrm{CH}), 2.33$ (br. s., $\left.6 \mathrm{H}, \mathrm{CH}_{3}\right) ;{ }^{13} \mathrm{C}-\mathrm{NMR}(126 \mathrm{MHz}, \mathrm{DMSO}$ $\left.d_{6}\right) \delta: 152.1,150.5,146.3,134.7,129.0,125.7,125.2,120.6$, 118.7, 118.4, 117.5, 33.0; ESI-MS m/z 497.8 [M] ${ }^{+}$.

4,4'-[(3-Hydroxy-4-methoxyphenyl)methylene] bis(3-methyl-1-phenyl-1H-pyrazol-5-ol) 3h Yield 91\% as a white solid; mp $200.0-202.0{ }^{\circ} \mathrm{C}$ [Lit. 201-203 [44]]; ${ }^{1} \mathrm{H}-\mathrm{NMR}\left(500 \mathrm{MHz}, D M S O-d_{6}\right) \delta: 13.90$ (br. s., $1 \mathrm{H}, \mathrm{OH}$ ), 12.38 (br. s., $1 \mathrm{H}, \mathrm{OH}), 8.82$ (br. s., $1 \mathrm{H}, \mathrm{OH}), 7.71$ (d, $J=8.23 \mathrm{~Hz}, 4 \mathrm{H}, \mathrm{Ar}-\mathrm{H}), 7.44(\mathrm{t}, J=7.68 \mathrm{~Hz}, 4 \mathrm{H}, \mathrm{Ar}-\mathrm{H})$, $7.24(\mathrm{t}, J=6.86 \mathrm{~Hz}, 2 \mathrm{H}, \mathrm{Ar}-\mathrm{H}), 6.79(\mathrm{~d}, J=8.78 \mathrm{~Hz}, 1 \mathrm{H}$, $\mathrm{Ar}-\mathrm{H}), 6.69$ (d, $J=1.65 \mathrm{~Hz}, 1 \mathrm{H}, \mathrm{Ar}-\mathrm{H}), 6.59$ (dd, $J=8.23$, $1.65 \mathrm{~Hz}, 1 \mathrm{H}, \mathrm{Ar}-\mathrm{H}), 4.83(\mathrm{~s}, 1 \mathrm{H}, \mathrm{CH}), 3.70(\mathrm{~s}, 3 \mathrm{H}$, $\mathrm{OCH}_{3}$ ), 2.30 (br. s., $6 \mathrm{H}, \mathrm{CH}_{3}$ ).

4, 4'- [(3,4-Dihydroxyphenyl)methylene] bis(3-methyl-1-phenyl-1H-pyrazol-5-ol) 3i Yield 93\% as a cream solid; mp $182.7-184.0{ }^{\circ} \mathrm{C}$; FTIR $\left(\mathrm{cm}^{-1}\right): 3206$ $(\mathrm{OH}), 1598(\mathrm{C}=\mathrm{N}), 1568(\mathrm{C}=\mathrm{C}), 1501(\mathrm{C}=\mathrm{C}), 1368(\mathrm{OH})$, $1291(\mathrm{OH}), 1191(\mathrm{C}-\mathrm{O}) ;{ }^{1} \mathrm{H}-\mathrm{NMR}\left(500 \mathrm{MHz}, \mathrm{DMSO}-d_{6}\right)$

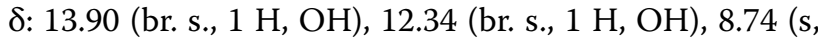
$1 \mathrm{H}, \mathrm{OH}), 8.60$ (br. s., $1 \mathrm{H}, \mathrm{OH}), 7.72(\mathrm{~d}, J=7.7 \mathrm{~Hz}, 4 \mathrm{H}$, $\operatorname{Ar}-\mathrm{H}), 7.44(\mathrm{t}, J=7.7 \mathrm{~Hz}, 4 \mathrm{H}, \mathrm{Ar}-\mathrm{H}), 7.24(\mathrm{t}, J=7.1 \mathrm{~Hz}$, $2 \mathrm{H}, \mathrm{Ar}-\mathrm{H}), 6.66(\mathrm{~d}, J=1.6 \mathrm{~Hz}, 1 \mathrm{H}, \mathrm{Ar}-\mathrm{H}), 6.61(\mathrm{~d}$, $J=8.2 \mathrm{~Hz}, 1 \mathrm{H}, \mathrm{Ar}-\mathrm{H}), 6.47(\mathrm{dd}, J=1.6,8.2 \mathrm{~Hz}, 1 \mathrm{H}$, $\mathrm{Ar}-\mathrm{H}$ ), 4.80 (s, $1 \mathrm{H}, \mathrm{CH}$ ), 2.29 (br. s., $\left.6 \mathrm{H}, \mathrm{CH}_{3}\right) ;{ }^{13} \mathrm{C}-\mathrm{NMR}$ $\left(126 \mathrm{MHz}, \mathrm{DMSO}-d_{6}\right) \delta: 146.2,144.8,143.4,132.9,128.9$, 125.5, 120.5, 117.8, 115.2, 114.8, 32.4, 11.6; HRMS (TOF $\mathrm{ES}+) \mathrm{m} / z$ calcd for $\mathrm{C}_{27} \mathrm{H}_{25} \mathrm{~N}_{4} \mathrm{O}_{4}(\mathrm{M}+\mathrm{H})^{+}:$469.1870; found: 469.1876 .

4,4'-[(4-Hydroxyphenyl)methylene]bis(3-methyl-1-phenyl-1H-pyrazol-5-ol) $3 j$ Yield $97 \%$ as a white solid; mp 217.2-218.9 ${ }^{\circ} \mathrm{C}$ [Lit. 214-216 [45]]; ${ }^{1} \mathrm{H}-\mathrm{NMR}(400 \mathrm{MHz}$, DMSO-d ${ }_{6}$ ) $\delta: 13.93$ (br. s., $\left.1 \mathrm{H}, \mathrm{OH}\right), 9.17$ (s, $\left.1 \mathrm{H}, \mathrm{OH}\right), 7.70$ $(\mathrm{d}, J=7.7 \mathrm{~Hz}, 4 \mathrm{H}, \mathrm{Ar}-\mathrm{H}), 7.43(\mathrm{t}, J=7.8 \mathrm{~Hz}, 4 \mathrm{H}, \mathrm{Ar}-\mathrm{H})$, $7.24(\mathrm{t}, J=7.3 \mathrm{~Hz}, 2 \mathrm{H}, A r-\mathrm{H}), 7.04(\mathrm{~d}, J=8.4 \mathrm{~Hz}, 2 \mathrm{H}$, $\mathrm{Ar}-\mathrm{H}), 6.65$ (d, $J=8.6 \mathrm{~Hz}, 2 \mathrm{H}, \mathrm{Ar}-\mathrm{H}), 4.84(\mathrm{~s}, 1 \mathrm{H}, \mathrm{CH})$, 2.29 (br. s., $6 \mathrm{H}, \mathrm{CH}_{3}$ ).

4,4'-[(4-Methoxyphenyl)methylene]bis(3-methyl-1-phenyl-1H-pyrazol-5-ol) $3 k$ Yield $92 \%$ as a white solid; mp 176.0-177.0 ${ }^{\circ} \mathrm{C}$ [Lit. 176-177 [46]]; ${ }^{1} \mathrm{H}-\mathrm{NMR}(400 \mathrm{MHz}$, DMSO-d ${ }_{6}$ ) $\delta: 13.92$ (br. s., $1 \mathrm{H}, \mathrm{OH}$ ), 12.39 (br. s., $1 \mathrm{H}$, $\mathrm{OH}), 7.70(\mathrm{~d}, J=7.9 \mathrm{~Hz}, 4 \mathrm{H}, \mathrm{Ar}-\mathrm{H}), 7.44(\mathrm{t}, J=7.3$, $7.9 \mathrm{~Hz}, 4 \mathrm{H}, \mathrm{Ar}-\mathrm{H}), 7.24(\mathrm{~d}, J=7.3 \mathrm{~Hz}, 2 \mathrm{H}, \mathrm{Ar}-\mathrm{H}), 7.16$ $(\mathrm{d}, J=8.5 \mathrm{~Hz}, 2 \mathrm{H}, \mathrm{Ar}-\mathrm{H}), 6.83(\mathrm{~d}, J=8.5 \mathrm{~Hz}, 2 \mathrm{H}, \mathrm{Ar}-\mathrm{H})$, 
4.89 (s, $1 \mathrm{H}, \mathrm{CH}$ ), 3.70 (s, $3 \mathrm{H}, \mathrm{OCH}_{3}$ ), 2.30 (br. s., $6 \mathrm{H}$, $\left.\mathrm{CH}_{3}\right)$.

4,4'-[(4-Nitrophenyl)methylene]bis(3-methyl-1-phenyl-1H-pyrazol-5-ol) $3 l$ Yield $97 \%$ as a yellow solid; mp 218.0-219.0 ${ }^{\circ} \mathrm{C}$ [Lit. 219-220 [47]]; ${ }^{1} \mathrm{H}-\mathrm{NMR}(500 \mathrm{MHz}$, $\left.D M S O-d_{6}\right) \delta: 8.17(\mathrm{~d}, J=8.9 \mathrm{~Hz}, 2 \mathrm{H}, \mathrm{Ar}-\mathrm{H}), 7.69$ (dd, $J=1.0,8.6 \mathrm{~Hz}, 4 \mathrm{H}, \mathrm{Ar}-\mathrm{H}), 7.51(\mathrm{~d}, J=8.9 \mathrm{~Hz}, 2 \mathrm{H}$, Ar-H), $7.45(\mathrm{t}, J=8.0 \mathrm{~Hz}, 4 \mathrm{H}, \mathrm{Ar}-\mathrm{H}), 7.26(\mathrm{t}, J=7.4 \mathrm{~Hz}$, $2 \mathrm{H}, \mathrm{Ar}-\mathrm{H}), 5.13(\mathrm{~s}, 1 \mathrm{H}, \mathrm{CH}), 2.34\left(\mathrm{~s}, 6 \mathrm{H}, \mathrm{CH}_{3}\right)$.

4,4'-[(4-Fluorophenyl)methylene]bis(3-methyl-1-phenyl-1H-pyrazol-5-ol) $3 m$ Yield $87 \%$ as a white solid; mp 183.8-185.8 ${ }^{\circ} \mathrm{C}$ [Lit. $181-183$ [48]]; ${ }^{1} \mathrm{H}-\mathrm{NMR}(500 \mathrm{MHz}$, DMSO- $\left.d_{6}\right) \delta: 7.59-7.65(\mathrm{~m}, 4 \mathrm{H}, \mathrm{Ar}-\mathrm{H}), 7.42(\mathrm{dd}, \mathrm{J}=8.3$, $7.4 \mathrm{~Hz}, 4 \mathrm{H}, \mathrm{Ar}-\mathrm{H}), 7.24(\mathrm{t}, \mathrm{J}=7.2 \mathrm{~Hz}, 4 \mathrm{H}, \mathrm{Ar}-\mathrm{H}), 7.06(\mathrm{t}$, $\mathrm{J}=8.9 \mathrm{~Hz}, 2 \mathrm{H}, \mathrm{Ar}-\mathrm{H}), 4.91(\mathrm{~s}, 1 \mathrm{H}, \mathrm{CH}), 2.27\left(\mathrm{~s}, 6 \mathrm{H}, \mathrm{CH}_{3}\right)$.

Methyl 4-[bis(5-hydroxy-3-methyl-1-phenyl-1H-pyrazol-4-yl)methyl]benzoate $3 n$ Yield quantitative as a white solid; mp $217.2-218.7{ }^{\circ} \mathrm{C}$, FTIR $\left(\mathrm{cm}^{-1}\right): 1720$ $(\mathrm{C}=\mathrm{O}), 1595(\mathrm{C}=\mathrm{N}), 1569(\mathrm{C}=\mathrm{C}), 1499(\mathrm{C}=\mathrm{C}), 1286$ (C-O), 1118 (C-O); ${ }^{1} \mathrm{H}-\mathrm{NMR}\left(400 \mathrm{MHz}, \mathrm{CDCl}_{3}\right.$ ) 8: 7.89 (d, $J=8.2 \mathrm{~Hz}, 2 \mathrm{H}, \mathrm{Ar}-\mathrm{H}), 7.60(\mathrm{~d}, J=7.9 \mathrm{~Hz}, 4 \mathrm{H}, \mathrm{Ar}-\mathrm{H})$, 7.29 (t, $J=7.7 \mathrm{~Hz}, 4 \mathrm{H}, \mathrm{Ar}-\mathrm{H}), 7.26(\mathrm{~d}, J=7.6 \mathrm{~Hz}, 2 \mathrm{H}$, Ar-H), 7.12 (t, J=7.4 Hz, 2H, Ar-H), $4.80(\mathrm{~s}, 1 \mathrm{H}, \mathrm{CH})$, $3.86\left(\mathrm{~s}, 3 \mathrm{H}, \mathrm{COOCH}_{3}\right), 2.12\left(\mathrm{~s}, 6 \mathrm{H}, \mathrm{CH}_{3}\right) ;{ }^{13} \mathrm{C}-\mathrm{NMR}$ $\left(101 \mathrm{MHz}, \mathrm{CDCl}_{3}\right)$ 8: 167.2, 146.8, 146.1, 129.9, 129.1, 128.5, 128.2, 128.1, 127.5, 126.4, 121.4, 52.2, 34.1, 12.0; ESI-MS m/z $494.8[\mathrm{M}]^{+}$.

4,4'-[(4-Trifluoromethylphenyl)methylene] bis(3-methyl-1-phenyl-1H-pyrazol-5-ol) 3o Yield 96\% as a white solid; mp $203.0-205.0{ }^{\circ} \mathrm{C}$; FTIR $\left(\mathrm{cm}^{-1}\right): 1600$ $(\mathrm{C}=\mathrm{N}), 1583(\mathrm{C}=\mathrm{C}), 1500(\mathrm{C}=\mathrm{C}), 1320\left(\mathrm{CF}_{3}\right), 1115$ $\left(\mathrm{CF}_{3}\right) ;{ }^{1} \mathrm{H}-\mathrm{NMR}\left(400 \mathrm{MHz}, \mathrm{DMSO}-d_{6}\right) \delta: 13.88(\mathrm{~s}, 1 \mathrm{H}$, $\mathrm{OH}), 7.70(\mathrm{~d}, J=7.7 \mathrm{~Hz}, 4 \mathrm{H}, \mathrm{Ar}-\mathrm{H}), 7.65(\mathrm{~d}, J=8.3 \mathrm{~Hz}$, $2 \mathrm{H}, \mathrm{Ar}-\mathrm{H}), 7.46(\mathrm{~d}, J=8.4 \mathrm{~Hz}, 2 \mathrm{H}, \mathrm{Ar}-\mathrm{H}), 7.44(\mathrm{t}$, $J=8.1 \mathrm{~Hz}, 4 \mathrm{H}, \mathrm{Ar}-\mathrm{H}), 7.25(\mathrm{t}, J=7.3 \mathrm{~Hz}, 2 \mathrm{H}, \mathrm{Ar}-\mathrm{H})$, $5.06(\mathrm{~d}, J=8.4 \mathrm{~Hz}, 1 \mathrm{H}, \mathrm{CH}), 2.33\left(\mathrm{~s}, 6 \mathrm{H}, \mathrm{CH}_{3}\right) ;{ }^{13} \mathrm{C}-\mathrm{NMR}$ $\left(101 \mathrm{MHz}, \mathrm{DMSO}_{\mathrm{d}}\right) \delta$ : 147.1, 146.3, 128.9, 128.1, 126.7 $(\mathrm{q}, J=31.5 \mathrm{~Hz}), 125.7(\mathrm{~m}), 125.1(\mathrm{q}, J=4.0 \mathrm{~Hz}), 124.5(\mathrm{q}$, $J=271.9 \mathrm{~Hz}), 120.6,33.0,11.6 ;{ }^{19} \mathrm{~F}-\mathrm{NMR}(56.17 \mathrm{MHz}$, DMSO-d 6 ) $\delta:-60.12\left(\mathrm{~s}, \mathrm{CF}_{3}\right) ; \mathrm{ESI}-\mathrm{MS} \mathrm{m} / \mathrm{z} 504.8[\mathrm{M}]^{+}$.

4,4'-[(4-Trifluoromethoxyphenyl)methylene] bis(3-methyl-1-phenyl-1H-pyrazol-5-ol) $3 p$ Yield quantitative as a white solid; $\mathrm{mp} 174.5-176.0^{\circ} \mathrm{C}$; FTIR $\left(\mathrm{cm}^{-1}\right)$ : $1596(\mathrm{C}=\mathrm{N}), 1579(\mathrm{C}=\mathrm{C}), 1501(\mathrm{C}=\mathrm{C}), 1253\left(\mathrm{C}-\mathrm{OCF}_{3}\right)$, $1226\left(\mathrm{CF}_{3}\right), 1167\left(\mathrm{CF}_{3}\right) ;{ }^{1} \mathrm{H}-\mathrm{NMR}\left(500 \mathrm{MHz}, \mathrm{DMSO}-d_{6}\right)$ $\delta: 7.70(\mathrm{~d}, J=7.8 \mathrm{~Hz}, 4 \mathrm{H}, \mathrm{Ar}-\mathrm{H}), 7.44(\mathrm{t}, J=7.8 \mathrm{~Hz}, 4 \mathrm{H}$, Ar-H), 7.35 (d, $J=8.6 \mathrm{~Hz}, 2 \mathrm{H}, \mathrm{Ar}-\mathrm{H}), 7.27$ (d, $J=8.5 \mathrm{~Hz}$, $2 \mathrm{H}, \mathrm{Ar}-\mathrm{H}), 7.25(\mathrm{t}, J=7.3 \mathrm{~Hz}, 2 \mathrm{H}, \mathrm{Ar}-\mathrm{H}), 5.00(\mathrm{~s}, 1 \mathrm{H}$,
$\mathrm{CH}), 2.32$ (br. s., $\left.6 \mathrm{H}, \mathrm{CH}_{3}\right) ;{ }^{13} \mathrm{C}$ NMR (126 MHz, DMSO$\left.d_{6}\right) \delta: 147.1,146.8,141.9,137.4,137.2,129.5,129.4,126.4$, 121.2, 33.0, $11.8 ;{ }^{19} \mathrm{~F}-\mathrm{NMR}\left(56.17 \mathrm{MHz}, \mathrm{DMSO}_{-} d_{6}\right) \delta$ : $-56.02\left(\mathrm{~s}, \mathrm{OCF}_{3}\right) ; \mathrm{ESI}-\mathrm{MS} \mathrm{m} / \mathrm{z} 520.8[\mathrm{M}]^{+}$.

4,4'-[(4-Thiomethylphenyl)methylene]bis(3-methyl-1-phenyl-1H-pyrazol-5-ol) $3 q$ Yield $60 \%$ as a white solid; mp 209.1-211.3 ${ }^{\circ} \mathrm{C}$ [Lit. 205-207 [49]]; ${ }^{1} \mathrm{H}-\mathrm{NMR}(400 \mathrm{MHz}$, $\left.D M S O-d_{6}\right) \delta: 13.91$ (br. s., $\left.1 \mathrm{H}, \mathrm{OH}\right), 7.70(\mathrm{~d}, J=7.9 \mathrm{~Hz}$, $4 \mathrm{H}, \mathrm{Ar}-\mathrm{H}), 7.44(\mathrm{t}, J=7.8 \mathrm{~Hz}, 4 \mathrm{H}, \mathrm{Ar}-\mathrm{H}), 7.24(\mathrm{t}$, $J=7.3 \mathrm{~Hz}, 2 \mathrm{H}, \mathrm{Ar}-\mathrm{H}), 7.18(\mathrm{~s}, 4 \mathrm{H}, \mathrm{Ar}-\mathrm{H}), 4.91(\mathrm{~s}, 1 \mathrm{H}$, $\mathrm{CH}$ ), 2.42 (s, $3 \mathrm{H}, \mathrm{SCH}_{3}$ ), 2.31 (br. s., $6 \mathrm{H}, \mathrm{CH}_{3}$ ).

\section{Biological evaluation}

\section{DPPH radical scavenging assay}

The stock solutions of the compounds was prepared by dissolving $3 \mathbf{a}-\mathbf{q}$ in dimethylsulfoxide (DMSO) to a concentration of $4 \mathrm{mg} / \mathrm{mL}$. The solution was, diluted with methanol until a concentration of $400 \mu \mathrm{g} / \mathrm{mL}$ was obtained and then used immediately.

The experimental procedure was adapted from the literature [50]. Briefly, $100 \mu \mathrm{L}$ of a $0.2 \mathrm{mM}$ methanol solution of DPPH (2, 2-diphenyl-1-picrylhydrazyl) radical were added to $100 \mu \mathrm{L}$ of methanolic solutions of $3 \mathbf{a}-\mathbf{q}$ prepared as serial two-fold dilutions from the stock solution in 96-well microfilter plates. Standards and edaravone were also prepared in the same concentrations. The mixture was incubated in dark at room temperature for $30 \mathrm{~min}$ and the absorbance was read at $515 \mathrm{~nm}$ on a Cytattion 5 (BioTek) spectrophotometer.

The \% DPPH scavenging activity was then calculated by using the following formula:

\%DPPH scavenging

$$
=100 *\left[\frac{\left(\mathrm{A}_{\text {sample }+\mathrm{DPPH}}-\mathrm{A}_{\text {sample blank }}\right)}{\left(\mathrm{A}_{\mathrm{DPPH}}-\mathrm{A}_{\text {solvent }}\right)}\right]
$$

The antioxidant activity of the compound was expressed as $\mathrm{IC}_{50}$, which is defined as the concentration that could scavenge $50 \%$ of the DPPH free radical. The $\mathrm{IC}_{50}$ values were calculated in GraphPad Prism 8.1.1 (GraphPad Software, Corp.) The results are given as a mean \pm standard deviation (SD) of experiments done in triplicate.

\section{Cell culture}

For biological studies, a human colorectal carcinoma RKO cell line (donated by Dra. Patricia Ostrosky, Instituto de Investigaciones Biomédicas, Departamento de Genética y Toxicología Ambiental, UNAM), with wild type p53 was used. Cells were cultured in RPMI-1640 medium supplemented with FBS 10\% (Sigma Aldrich, 
USA), glutamine $2 \mathrm{mM}$ (GIBCO-Thermo Fisher Scientific, USA), streptomycin $0.1 \mathrm{mg} / \mathrm{mL}$, penicillin $100 \mathrm{U} /$ $\mathrm{mL}$, and amphotericin B $0.25 \mu \mathrm{g} / \mathrm{mL}$; and maintained at $37{ }^{\circ} \mathrm{C}$ in a humidified atmosphere containing $5 \% \mathrm{CO}_{2}$. Derivatives were dissolved in DMSO at a stock concentration of $20 \mathrm{mM}$. The final concentration of DMSO $(<1 \%, v / v)$ did not affect the cell growth in the different experiments performed.

\section{Cytotoxic assay}

The effect of each compound on cell proliferation was evaluated by the MTS metabolic viability assay, measuring mitochondrial activity of live cells. For this purpose, $2 \times 10^{3}$ cells in $100 \mu \mathrm{L}$ per well were seeded in triplicate in 96-well plates. Twenty-four hours after seeding, cells were exposed to each one of the derivatives at increasing concentrations $(5-250 \mu \mathrm{M})$ and incubated for $48 \mathrm{~h}$. Later, $20 \mu \mathrm{L}$ of Cell Titer 96 Aqueous One Solution cell proliferation reagent (Promega, USA) was added to each well containing the cells $4 \mathrm{~h}$ before finishing the treatment. Then, the absorbance was measured with a microplate spectrophotometer (Epoch 2-BioTek, USA) at $492 \mathrm{~nm}$. Data obtained from untreated cells (control) were considered as $100 \%$ of the viability to normalize the absorbance of treated samples.

Trypan Blue dye exclusion assay was also performed to determine the cell number and viability after exposure to compound 3i. Briefly: $3 \times 10^{4}$ cells in $2 \mathrm{~mL}$ per well were seeded in a 6-well plate; after $24 \mathrm{~h}$, cells were exposed to compound $3 \mathbf{i}$ at $10-70 \mu \mathrm{M}$ and incubated for additional 24 and $48 \mathrm{~h}$. Supernatant from wells was recovered independently, cells were then trypsinized, collected, and mixed with the previously recovered medium. After centrifugation, pellets were re-suspended in $1 \mathrm{~mL}$ of fresh medium. Cell suspension was mixed with Trypan Blue 0.4\% (GIBCO-Thermo Fisher Scientific, USA) in a 1:1 proportion and then counted applying a hemocytometer. Viable and non-viable (stained) cells were counted in a light microscopy (Nikon, USA).

For morphological analysis, $5 \times 10^{4}$ cells $/ \mathrm{mL}$ were seeded in $3.5-\mathrm{cm}$ diameter Petri dishes. After $24 \mathrm{~h}$ of incubation, cells were exposed to compound $3 \mathbf{i}$ at three representative doses: 30,40 and $50 \mu \mathrm{M}$, for 24 and $48 \mathrm{~h}$. Subsequently, cell morphology was observed using a light microscope (Axioskop 2 plus-Zeiss, Germany) equipped with a $40 \times$ objective. Images were then acquired with a SCA1300-32FM digital camera (Basler Inc., Germany).

\section{Western blot analysis}

Western blot analyses were performed in order to determine the induced cell death pathway by derivatives. According to cytotoxic effect, three concentrations (30, 40 and $50 \mu \mathrm{M}$ ) were administrated to RKO cell line for
$24 \mathrm{~h}$. As positive controls, cells were simultaneously exposed for $10 \mathrm{~min}$ to UV irradiation (Osram, G30T8, $30 \mathrm{~W}$ Germicidal UV-C Lamp, $254 \mathrm{~nm}$ ) for apoptosis induction or for $1 \mathrm{~h}$ to PBS for starvation-induced autophagy [51]. As follows, proteins were separated by SDS-PAGE (7-15\%) and transferred to a PVDF membrane (IPVH00010, Immobilon-P, $0.45 \mu \mathrm{m}$, EMD/Millipore, Billerica, USA), and then incubated with primary antibodies: p53 (sc-81168), p21 (sc-817), SQSTM1/ p62 (sc-48402), $\beta$-actin (sc-58673) (Santa Cruz Biotechnology, USA), PARP (\#9542), caspase-3 (\#6962), Bax (\#2774), Bcl-2 (\#15071) LC3A/B (\#12741) (Cell Signaling Technology, USA), followed by secondary antibodies: anti-mouse IgG, HRP-linked (\#7076) and anti-rabbit IgG, HRP-linked (\#7074) (Cell Signaling Technology, USA). Immunoreactive bands were monitored using Immobilon Crescendo, or Forte, Western HRP Substrate (MilliporeMerck, KGaA, Germany).

\section{Statistical analysis}

Each experiment was performed independently at least three times and data were reported as the means \pm SEM, as evidenced in each figure. Significant data were obtained with one-way analysis of variance (ANOVA) followed by the Dunnett post-test. Samples exposed to derivatives, Doxorrubicin or UV or starvation condition were compared to the control considering a $p<0.05$ to be statistically significant. Statistical analyses were realized in GraphPad Prism 8 (GraphPad Software, USA).

\section{Abbreviations}

FTIR: Fourier-transform infrared; NMR: Nuclear magnetic resonance; DPPH: 2,2-Diphenyl-1-picryl-hydrazyl-hydrate; RKO: Human colorectal carcinoma cell line; ALS: Amyotrophic lateral sclerosis; NaOAc: Sodium acetate; EtOH: Ethanol; $\mathrm{IC}_{50}$ : Half maximal inhibitory concentration/median inhibitory concentration; HM: Micromolar; Mp: Melting point; min: Minutes; Quant: Quantitative; MTS: 3-(4,5-Dimethylthiazol-2-yl)-2,5-diphenyltetrazolium bromide; p53: Tumor protein p53; p21: Cyclin-dependent kinase inhibitor 1; A2780: Human ovarian adenocarcinoma cell line; P388: Human leukemia cell line; A549: Human lung carcinoma cell line; LC3 II: Microtubule-associated protein 1A/1B-light chain 3; LC3 I: Cytosolic form of LC3; p62: Ubiquitin-binding protein p62; Bax: BCl-2-Like Protein 4; BCl-2: B-cell lymphoma 2; PARP-1: Poly [ADP-ribose] polymerase 1; ATR: Attenuated total refection; DMSO: Dimethyl sulfoxide; TMS: Tetramethylsilane; TOF: Time of flight; TLC: Thin layer chromatography; UV: Ultraviolet; HRMS: High-resolution mass spectrometry; ES+: Positive electrospray ionization; RPMI: Cell culture media Roswell Park Memorial Institute; FBS: Fetal bovine serum; PBS: Phosphate-buffered saline; SDS-PAGE: Sodium dodecyl sulphatepolyacrylamide gel electrophoresis; PVDF: Polyvinylidene difluoride; SQSTM1/ p62: Sequestosome-1; IgG: Immunoglobulin G; HRP: Horseradish peroxidase; SEM: Standard error of the mean.

\section{Supplementary Information}

The online version contains supplementary material available at https://doi. org/10.1186/s13065-021-00765-y.

Additional file 1: Fig. S1. Original western blots CL-X Posure ${ }^{\mathrm{TM}}$ films for Western blots. Each protein was detected in independent films at different times of exposure to the membrane. A) p53; B) p21; C) L(C3-I and -I; D) 
p62; E) BAX; F) BCl-2; G) Cleaved caspase-3; H) Active and cleaved PARP-1; I) Actin. Notice, for autophagy detection starvation control was first, in contrast to apoptosis control, which UV control was first. Fig. S2. ${ }^{1}$ H NMR spectrum of compound $\mathbf{3 a}$. Fig. S3. ${ }^{1} \mathrm{H}$ NMR spectrum of compound $\mathbf{3} \mathbf{b}$. Fig. S4. ${ }^{1} H$ NMR spectrum of compound $3 \mathbf{3 c}$. Fig. S5. ${ }^{1} H$ NMR spectrum of compound 3d. Fig. S6. ${ }^{1} \mathrm{H}$ NMR spectrum of compound 3e. Fig. S7. ${ }^{19} \mathrm{~F}$ NMR spectrum of compound 2e. Fig. S8. ${ }^{1} \mathrm{H}$ NMR spectrum of compound 3f. Fig. S9. ${ }^{1} \mathrm{H}$ NMR spectrum of compound $\mathbf{3}$ g. Fig. $\mathbf{S 1 0} .{ }^{13} \mathrm{C}$ NMR spectrum of compound $\mathbf{3} \mathbf{~ g}$. Fig. S11. FTIR spectrum of compound $\mathbf{3} \mathbf{~ g}$. Fig. S12. ESI-MS spectrum of compound $\mathbf{3} \mathbf{~ g}$. Fig. S13. ${ }^{1} \mathrm{H}$ NMR spectrum of compound $\mathbf{3}$ h. Fig. S14. ${ }^{1} \mathrm{H}$ NMR spectrum of compound $\mathbf{3 i}$. Fig. S15. ${ }^{13} \mathrm{C}$ NMR spectrum of compound 3i. Fig. S16. FTIR spectrum of compound 3i. Fig. S17. HRMS spectrum of compound 3i. Fig. S18. ${ }^{1} H$ NMR spectrum of compound $\mathbf{3 j}$. Fig. S19. ${ }^{1} \mathrm{H}$ NMR spectrum of compound $\mathbf{3} \mathbf{k}$. Fig. S20. ${ }^{1} H$ NMR spectrum of compound $\mathbf{3} \mathbf{I}$. Fig. S21. ${ }^{1} \mathrm{H}$ NMR spectrum of compound $\mathbf{3} \mathbf{~}$. Fig. $\mathbf{S 2 2} .{ }^{19} \mathrm{~F}$ NMR spectrum of compound $\mathbf{3} \mathbf{~} \mathbf{\text { . Fig. }}$ S23. ${ }^{1} \mathrm{H}$ NMR spectrum of compound $\mathbf{3 n}$. Fig. S24. ${ }^{13} \mathrm{C}$ NMR spectrum of compound 3n. Fig. S25. FTIR spectrum of compound 3n. Fig. S26. ESIMS spectrum of compound 3 n. Fig. S27. ${ }^{1} H$ NMR spectrum of compound 3o. Fig. S28. ${ }^{13} \mathrm{C}$ NMR spectrum of compound 3o. Fig. S29. ${ }^{19} \mathrm{~F}$ NMR spectrum of compound $\mathbf{3 0}$. Fig. S30. FTIR spectrum of compound $\mathbf{3 0}$. Fig. S31. ESI-MS spectrum of compound 3o. Fig. S32. ${ }^{1} \mathrm{H}$ NMR spectrum of compound $\mathbf{3 p}$. Fig. S33. ${ }^{13} \mathrm{C}$ NMR spectrum of compound $\mathbf{3 p}$. Fig. S34. ${ }^{19} \mathrm{~F}$ NMR spectrum of compound 3p. Fig. S35. FTIR spectrum of compound 3p. Fig. S36. ESI-MS spectrum of compound 3p. Fig. S37. ${ }^{1} \mathrm{H}$ NMR spectrum of compound $\mathbf{3 q}$.

\section{Acknowledgements}

The authors express their sincere thanks to Dr. John R. Lloyd and Dr. Robert O'Connor at NIDDK-NIH and Joshua J. Kellogg at Department of Chemistry \& Biochemistry, University of North Carolina Greensboro, for providing HRMS and NMR spectra. Dr. Kenneth L. Kirk (NIDDK-NIH) provided helpful comments.

\section{Authors' contributions}

JECC, JCRB and JHM carried out the synthesis and characterization experiments. JHM performed the DPPH assay. KEMS, NVOG, LMGO, NBM and JCRB performed the biological activities. JCRB and JHM designed and performed the research, analyzed the data, interpreted the results and prepared the manuscript. All authors read and approved the final manuscript.

\section{Funding}

This work was supported by Corporación Ecuatoriana para el Desarrollo de la Investigación y la Academia (CEDIA), (CEPRA XI-2017-10), Universidad Técnica Particular de Loja (UTPL), (PROY_INV_QUI_2017_2222), Universidad UTE, and Universidad Central del Ecuador.

\section{Availability of data and materials}

All data generated or analysed during this study are included in this published article [and its additional information file].

\section{Declarations}

Ethics approval and consent to participate

Not applicable.

\section{Consent for publication}

Not applicable.

\section{Competing interests}

The authors declare that they have no competing interests.

\section{Author details}

${ }^{1}$ Facultad de Ciencias Químicas, Universidad Central del Ecuador, Quito, Ecuador. ${ }^{2}$ Departamento de Ciencias de La Salud, Universidad Técnica Particular de Loja, San Cayetano Alto s/n, C.P. 1101 608, Loja, Ecuador. ${ }^{3}$ Departamento de Química y Ciencias Exactas, Universidad Técnica Particular de Loja, San Cayetano Alto s/n, C.P. 1101 608, Loja, Ecuador. ${ }^{4}$ Centro de Investigación
Biomédica (CENBIO), Facultad de Ciencias de la Salud Eugenio Espejo, Universidad UTE, 170527 Quito, Ecuador.

Received: 8 March 2021 Accepted: 19 May 2021

Published online: 03 June 2021

\section{References}

1. Gomtsyan A (2012) Heterocycles in drugs and drug discovery. Chem Heterocycl Compd 48:7-10. https://doi.org/10.1007/s10593-012-0960-z

2. Pandey A, Dewangan D, Verma S et al (2011) Synthesis of Schiff bases of 2-amino-5-aryl-1, 3, 4-thiadiazole And its Analgesic, Anti- Inflammatory, Anti-Bacterial and Anti-Tubercular Activity. Int J ChemTech Res 3:178-184

3. Halim KNM, Ramadan SK, Rizk SA, El-Hashash MA (2020) Synthesis, DFT study, molecular docking and insecticidal evaluation of some pyrazolebased tetrahydropyrimidine derivatives. Synth Commun 50:1159-1175. https://doi.org/10.1080/00397911.2020.1720739

4. Naim MJ, Alam O, Nawaz F et al (2016) Current status of pyrazole and its biological activities. J Pharm Bioallied Sci 8:2-17. https://doi.org/10.4103/ 0975-7406.171694

5. Mohamed MS, Abdelhamid AO, Almutairi FM et al (2018) Induction of apoptosis by pyrazolo[3,4-d]pyridazine derivative in lung cancer cells via disruption of $\mathrm{BCl}-2 / \mathrm{Bax}$ expression balance. Bioorg Med Chem 26:623-629. https://doi.org/10.1016/j.bmc.2017.12.026

6. Ramadan SK, Halim KNM, Rizk SA, El-Hashash MA (2020) Cytotoxic activity and density functional theory studies of some 1,3-diphenylpyrazolyltetrahydropyrimidine derivatives. J Iran Chem Soc 17:1575-1589. https://doi. org/10.1007/s13738-020-01880-8

7. Ramadan SK, El-Ziaty AK, Ali RS (2021) Synthesis, antiproliferative activity, and molecular docking of some $\mathrm{N}$-heterocycles bearing a pyrazole scaffold against liver and breast tumors. J Heterocycl Chem 58:290-304. https://doi.org/10.1002/jhet.4168

8. Halim KNM, Rizk SA, El-Hashash MA, Ramadan SK (2021) Straightforward synthesis, antiproliferative screening, and density functional theory study of some pyrazolylpyrimidine derivatives. J Heterocycl Chem 58:636-645. https://doi.org/10.1002/jhet.4204

9. Bailly C, Hecquet PE, Kouach M et al (2020) Chemical reactivity and uses of 1-phenyl-3-methyl-5-pyrazolone (PMP), also known as edaravone. Bioorganic Med Chem 28:1-8. https://doi.org/10.1016/j.bmc.2020.115463

10. Higashi Y, Jitsuiki D, Chayama K, Yoshizumi M (2006) Edaravone (3-methyl1-phenyl-2-pyrazolin-5-one), a novel free radical scavenger, for treatment of cardiovascular diseases. Recent Pat Cardiovasc Drug Discov 1:85-93. https://doi.org/10.2174/157489006775244191

11. Yamamoto I, Azuma Y, Yamaguchi M (2019) Cancer-related genes and ALS. Front Biosci 24:1241-1258. https://doi.org/10.2741/4777

12. Murias M, Jäger W, Handler N et al (2005) Antioxidant, prooxidant and cytotoxic activity of hydroxylated resveratrol analogues: Structure-activity relationship. Biochem Pharmacol 69:903-912. https://doi.org/10.1016/j. bcp.2004.12.001

13. Grigalius I, Petrikaite V (2017) Relationship between antioxidant and anticancer activity of trihydroxyflavones. Molecules 22:2169. https://doi. org/10.3390/molecules22122169

14. Hamama WS (2001) Pyrazolones as versatile precursors for the synthesis of fused and binary heterocycles. Synth Commun 31:1335-1345. https:// doi.org/10.1081/SCC-100104042

15. Li X-L, Wang Y-M, Tian B et al (1998) The solid-state michael addition of 3-methyl-1-phenyl-5-pyrazolone. J Heterocycl Chem 35:129-134. https:// doi.org/10.1002/jhet.5570350124

16. Hasaninejad A, Kazerooni MR, Zare A (2013) Room-temperature, catalystfree, one-pot pseudo-five-component synthesis of 4,4-(arylmethylene) bis(3-methyl-1-phenyl-1H-pyrazol-5-ol)s under ultrasonic irradiation. ACS Sustain Chem Eng 1:679-684. https://doi.org/10.1021/sc400081c

17. Noruzian F, Olyaei A, Hajinasiri R, Sadeghpour M (2019) Guanidine hydrochloride catalyzed efficient one-pot pseudo five-component synthesis of 4,4'-(arylmethylene)bis(1 H-pyrazol-5-ols) in water. Synth Commun 49:2717-2724. https://doi.org/10.1080/00397911.2019.1643483

18. Moosavi-Zare AR, Zolfigol MA, Noroozizadeh E et al (2016) Cyclocondensation-Knoevenagel-Michael Domino reaction of phenyl hydrazine, acetoacetate derivatives and aryl aldehydes over acetic acid functionalized 
ionic liquid. Res Chem Intermed 42:4759-4772. https://doi.org/10.1007/ s11164-015-2317-6

19. Kauthale SS, Tekale SU, Jadhav KM, Pawar RP (2016) Ethylene glycol promoted catalyst-free pseudo three-component green synthesis of bis(coumarin)s and bis(3-methyl-1-phenyl-1 $\mathrm{H}$-pyrazol-5-ol)s. Mol Divers 20:763-770. https://doi.org/10.1007/s11030-016-9673-z

20. Diwan F, Shaikh M, Farooqui M (2018) Lemon juice catalyzed efficient one-pot synthesis, antioxidant and antimicrobial evaluation of bispyrazolyl methanes. Chem Biol Interface 8:255-268

21. Mahajan PS, Nikam MD, Khedkar V et al (2017) An Organocatalyzed efficient one-pot synthesis, biological evaluation, and molecular docking studies of 4,4'-(arylmethylene)bis-(3-methyl-1-phenyl-1 H-pyrazol-5-ols). J Heterocycl Chem 54:1109-1120. https://doi.org/10.1002/jhet.2681

22. Yang $X$, Zhang $P$, Zhou $Y$ et al (2012) Synthesis and Antioxidant Activities of Novel 4,4'-Arylmethylene-bis $(1 \mathrm{H}$-pyrazole-5-ol)s from Lignin. Chinese J Chem 30:670-674. https://doi.org/10.1002/cjoc.201280009

23. Bhavanarushi S, Kanakaiah V, Bharath G et al (2014) Synthesis and antibacterial activity of 4,4'-(aryl or alkyl methylene)-bis(1H-pyrazol5-ol) derivatives. Med Chem Res 23:158-167. https://doi.org/10.1007/ s00044-013-0623-3

24. Wang LF, Zhang HY (2003) A theoretical investigation on DPPH radicalscavenging mechanism of edaravone. Bioorg Med Chem Lett 13:37893792. https://doi.org/10.1016/j.bmcl.2003.07.016

25. Borges RS, Queiroz AN, Mendes APS et al (2012) Density Functional theory (DFT) study of edaravone derivatives as antioxidants. Int J Mol Sci 13:7594-7606. https://doi.org/10.3390/ijms13067594

26. Queiroz AN, Mendes APS, Leal MS et al (2010) Tautomerism and radicalscavenging activity of edaravone by DFT methods. J Comput Theor Nanosci 7:153-156. https://doi.org/10.1166/jctn.2010.1339

27. Foti M, Piattelli M, Baratta MT, Ruberto G (1996) Flavonoids, coumarins, and cinnamic acids as antioxidants in a micellar system. structure-activity relationship. J Agric Food Chem 44:497-501. https://doi.org/10.1021/ jf950378u

28. Wright JS, Johnson ER, DiLabio GA (2001) Predicting the activity of phenolic antioxidants: theoretical method, analysis of substituent effects, and application to major families of antioxidants. J Am Chem Soc 123:11731183. https://doi.org/10.1021/ja002455u

29. Collavin L, Lunardi A, Del Sal G (2010) P53-family proteins and their regulators: hubs and spokes in tumor suppression. Cell Death Differ 17:901-911. https://doi.org/10.1038/cdd.2010.35

30. Galluzzi L, Vitale I, Aaronson SA et al (2018) Molecular mechanisms of cell death: recommendations of the nomenclature committee on cell death 2018. Cell Death Differ 25:486-541. https://doi.org/10.1038/ s41418-017-0012-4

31. Polkam N, Ramaswamy VR, Rayam P et al (2016) Synthesis, molecular properties prediction and anticancer, antioxidant evaluation of new edaravone derivatives. Bioorg Med Chem Lett 26:2562-2568. https://doi. org/10.1016/j.bmcl.2016.03.024

32. Marković $\mathrm{V}$, Erić $\mathrm{S}$, Juranić ZD et al (2011) Synthesis, antitumor activity and QSAR studies of some 4-aminomethylidene derivatives of edaravone. Bioorg Chem 39:18-27. https://doi.org/10.1016/j.bioorg.2010.10.003

33. Zheng LW, LiY, Ge D et al (2010) Synthesis of novel oxime-containing pyrazole derivatives and discovery of regulators for apoptosis and autophagy in A549 lung cancer cells. Bioorganic Med Chem Lett 20:4766-4770. https://doi.org/10.1016/j.bmcl.2010.06.121

34. Saito T, Kuma A, Sugiura Y et al (2019) Autophagy regulates lipid metabolism through selective turnover of NCoR1. Nat Commun 10:1567. https:// doi.org/10.1038/s41467-019-08829-3

35. Guamán-Ortiz LM, Bailon-Moscoso N, Morocho V et al (2019) Onoseriolide, from Hedyosmum racemosum, induces cytotoxicity and apoptosis in human colon cancer cells. Nat Prod Res. https://doi.org/10.1080/14786 419.2019.1690485

36. Giansanti V, Torriglia A, Scovassi Al (2011) Conversation between apoptosis and autophagy: Is it your turn or mine? Apoptosis 16:321-333. https:// doi.org/10.1007/s10495-011-0589-x

37. Du L, Fei Z, Song S, Wei N (2017) Antitumor activity of Lobaplatin against esophageal squamous cell carcinoma through caspase-dependent apoptosis and increasing the Bax/Bcl-2 ratio. Biomed Pharmacother 95:447-452. https://doi.org/10.1016/j.biopha.2017.08.119

38. Haque ME, Asanuma M, Higashi Y et al (2003) Apoptosis-inducing neurotoxicity of dopamine and its metabolites via reactive quinone generation in neuroblastoma cells. Biochim Biophys Acta Gen Subj 1619:39-52. https://doi.org/10.1016/S0304-4165(02)00440-3

39. Phatangare KR, Padalkar VS, Gupta VD et al (2012) Phosphomolybdic acid: an efficient and recyclable solid acid catalyst for the synthesis of 4,4'-(arylmethylene)bis(1 H-pyrazol-5-ols). Synth Commun 42:1349-1358. https://doi.org/10.1080/00397911.2010.539759

40. Zolfigol MA, Ayazi-Nasrabadi R, Baghery S (2015) Synthesis and characterization of two novel biological-based nano organo solid acids with urea moiety and their catalytic applications in the synthesis of $4,4^{\prime}$-(arylmethylene)bis(1 H-pyrazol-5-ol), coumarin-3-carboxylic acid and cinnamic ac. RSC Adv 5:71942-71954. https://doi.org/10.1039/C5RA14001C

41. Rezaei F, Amrollahi MA, Khalifeh R (2020) Brønsted acidic dicationic ionic liquid immobilized on Fe3O4@SiO2 nanoparticles as an efficient and magnetically separable catalyst for the synthesis of bispyrazoles. ChemistrySelect 5:1760-1766. https://doi.org/10.1002/slct.201904831

42. Khan KM, Muhammad MT, Khan I et al (2015) Rapid cesium fluoridecatalyzed Knoevenagel condensation for the synthesis of highly functionalized 4,4'-(arylmethylene)bis(1H-pyrazol-5-ol) derivatives. Monatshefte für Chemie - Chem Mon 146:1587-1590. https://doi.org/10.1007/ s00706-015-1424-9

43. Kuarm BS, Rajitha B (2012) Xanthan sulfuric acid: an efficient, biosupported, and recyclable solid acid catalyst for the synthesis of $4,4^{\prime}$-(arylmethylene)bis(1H-pyrazol-5-ols). Synth Commun 42:2382-2387. https:// doi.org/10.1080/00397911.2011.557516

44. Das Gupta A, Pal R, Mallik AK (2014) Two efficient and green methods for synthesis of 4,4'-(arylmethylene)bis(1 H-pyrazol-5-ols) without use of any catalyst or solvent. Green Chem Lett Rev 7:404-411. https://doi.org/10. 1080/17518253.2014.970236

45. Niknam K, Habibabad MS, Deris A, Aeinjamshid N (2013) Preparation of silica-bonded $\mathrm{N}$-propyltriethylenetetramine as a recyclable solid base catalyst for the synthesis of 4,4'-(arylmethylene)bis( $1 \mathrm{H}$ - pyrazol-5-ols). Monatsh Chem 144:987-992. https://doi.org/10.1007/s00706-012-0910-6

46. Sobhani S, Hasaninejad A-R, Maleki MF, Parizi ZP (2012) Tandem Knoevenagel-Michael reaction of 1-phenyl-3-methyl-5-pyrazolone with aldehydes using 3-aminopropylated silica gel as an efficient and reusable heterogeneous catalyst. Synth Commun 42:2245-2255. https://doi.org/ 10.1080/00397911.2011.555589

47. Sobhani S, Safaei E, Hasaninejad A-R, Rezazadeh S (2009) An eco-friendly procedure for the efficient synthesis of bis(indolyl)methanes in aqueous media. J Organomet Chem 694:3027-3031. https://doi.org/10.1016/j. jorganchem.2009.05.004

48. Bakherad M, Keivanloo A, Amin AH et al (2017) A rapid, easy, and efficient method for synthesis of 4,4'-(arylmethylene)-bis-(1H-pyrazol-5-ols), catalyzed by boehmite nanoparticles. J Appl Chem 11:31-37. https://doi.org/ 10.1007/s40092-014-0072-8

49. Masoumeh K, Vafaei-nezhad M (2016) Design and characterization of L-prolinate-amberlite as a novel heterogeneous organocatalyst and its catalytic application in the synthesis of pyrazol-derivates. Catal Letters 146:353-363. https://doi.org/10.1007/s10562-015-1668-3

50. Les F, Prieto JM, Arbonés-Mainar J et al (2015) Bioactive properties of commercialised pomegranate (Punica granatum) juice: antioxidant, antiproliferative and enzyme inhibiting activities. Food Funct. https://doi. org/10.1039/C5FO00426H

51. Guamán-Ortiz LM, Romero-Benavides JC, Suarez Al et al (2020) Cytotoxic property of grias neuberthii extract on human colon cancer cells: a crucial role of autophagy. Evid Based Complement Altern Med 2020:1-11. https://doi.org/10.1155/2020/1565306

\section{Publisher's Note}

Springer Nature remains neutral with regard to jurisdictional claims in published maps and institutional affiliations. 